\title{
Innovationsmanagement in kleinen mittelständischen Unternehmen - Ansatzpunkte für ein Forschungskonzept mit Beratungsoptionen
}

\author{
Prof. Dr. phil. Rainer Voß
}

\section{Kleine und mittlere Unternehmen - ein gewichtiger Wirtschaftsfaktor}

Kleine und mittlere Unternehmen (KMU) sind eine tragende Säule der deutschen Wirtschaft. Von den 1992 erfaßten mehr als 1,9 Millionen Unternehmen mit einem Umsatz ab 500 TDM waren 78,4\% kleine und 21,3\% mittlere Unternehmen (1 bis 100 Mio DM Umsatz). Nur 0,3\% aller Unternehmen wiesen einen Umsatz von mehr als 100 Mio DM aus und sind somit als große Unternehmen zu bezeichnen (Kaiser, 1996).

Ebenso beeindruckend sind die Beiträge, die diese KMU im Verarbeitenden Gewerbe zur Wirtschaftskraft leisten. Sie lagen für die alten Bundesländer im Jahre 1990 bei Anteilen zwischen 40 und $65 \%$ bezogen auf das Bruttosozialprodukt, den Gesamtumsatz der Industrie, die Gesamtinvestitionen und die Industriebeschäftigten. Der Anteil an der Ausbildungsleistung der Industrie betrug sogar 80\% (BMWi, 1994, S. 14).

Eine solche im Wettbewerb erbrachte Wirtschaftsleistung läßt erwarten, daß KMU auch eine entsprechende Innovationsintensität aufweisen, um ihre Wettbewerbsfähigkeit zu erhalten und auszubauen. Dafür einige Belege: Selbst die absoluten Anteile der KMU an den FuE-Beschäftigten aller Unternehmen sind mit fast $15 \%$ in Westdeutschland und mit annähernd 68\% in Ostdeutschland erheblich ${ }^{1}$ (Wölfling, 1995, S. 414).

Die FuE-Intensität ${ }^{2}$ ist bei KMU, insbesondere bei Unternehmen mit unter 100 Beschäftigten, wesentlich höher als bei großen Unternehmen. Bei ostdeutschen mittelständischen Unternehmen ist sie noch höher als bei westdeutschen KMU, in der Größenklasse unter 100 Beschäftigte sogar doppelt bzw. dreifach so hoch. Innerhalb dieser Klasse weisen die kleinsten und kleinen Unternehmen noch ein besonders starkes Engagement in der Forschung zur Sicherung ihres Innovationspotentials auf ${ }^{3}$ (Wölfling, 1995, S. 413).

KMU orientieren ihre FuE-Anstrengungen zu fast 50\% auf die Hervorbringung von Produkten und Verfahren, die zur sogenannten höherwertigen Technik ${ }^{4}$ und zu 30\% auf solche, die zur Spitzentechnik ${ }^{5}$ gezählt werden (SVWissenschaftsstatistik, 1995, S. 7). Auch hier gibt es branchenspezifische Unterschiede in der Orientierung der Innovationsaktivitäten von KMU u. a. je nach Wettbewerbsintensität und „Innovationsdomänen“ der großen Unternehmen.

Schließlich deuten auch auf den Produktlebenszyklus bezogene Analysen darauf hin, daß mittelständische Unternehmen eine hohe Innovationsneigung aufweisen. So hatten sie 1991 beispielsweise einen geringeren
Anteil von Produkten in der Schrumpfungsphase (15\%) als etwa Großunternehmen (25\%) und mehr in Einfuihrungs- bzw. Reifephasen (Penzkofer, 1992, S. 5).

Diese nicht nur quantitativ, sondern vor allem qualitativ besondere Rolle der KMU - und hier speziell der kleinen forschungsintensiven Unternehmen - für eine moderne, dynamische und wettbewerbsfähige Wirtschaft hat auch dazu gefuihrt, daß dem innovativen Mittelstand frühzeitig in den USA, seit längerem in Deutschland und zunehmend auch auf EU-Ebene (Grünbuch, 1995) besondere Aufmerksamkeit gewidmet wird.

Die Technologie- und Wirtschaftspolitik in Deutschland fördert seit vielen Jahren, mit vielfältigen, auch spezifischen Maßnahmen und Instrumenten, die Gründung und Entwicklung kleiner innovationsorientierter Unternehmen. ${ }^{6}$ Gemessen an den evtl. auch zu hoch gesetzten Erwartungen, werden die erzielten Wirkungen häufig als zu gering oder nur indirekt bzw. schwer nachweisbar eingeschätzt ( Meyer-Krahmer, 1989). Die meisten dieser Maßnahmen wurden direkt, oft als Neuauflage, auf die neuen Bundesländer uibertragen und hatten bzw. haben hier über einen bestimmten Zeitraum zum Teil erhebliche Anfangserfolge zu verzeichnen. Allerdings beginnen je nach Maßnahme und Zeitdauer ihrer Anwendung oft bereits ähnliche Probleme wie in den alten Bundesländern aufzutreten.

Als mögliche Ursachen für nicht ausreichende Wirkungen werden häufig genannt:

- die ungünstigen rechtlichen bzw. finanzierungsseitigen Rahmenbedingungen in Deutschland,

- die ungenügende Kenntnis der spezifischen Entwicklungsprobleme von innovationsorientierten KMU (Kulicke et al., 1993, S. 1f),

- ïberwiegend angebotsorientierte und technikzentrierte Fördermaßnahmen und -instrumente (Staudt, 1996).

Wesentliche Fortschritte sind in Deutschland, zumindest was die Förderung und Analyse von Entwicklungsbedingungen betrifft, bezogen auf die sogenannten technologieorientierten Unternehmen (TOU) erreicht worden. Hier wurde im Jahre 1983 ein spezielles Programm Modellversuch „Förderung technologieorientierter Unternehmensgründungen" mit dem Ziel aufgelegt, die Start- und Wachstumsbedingungen solcher Gründungen zu verbessern und Erkenntnisse über deren Chancen und Risiken zu gewinnen (Kulicke et al., 1993, S. 1). Dieses Programm lief bis 1988 und wurde 1990 für die neuen Bundesländer neu aufgelegt. 
In den alten Bundesländern werden jährlich etwa 300 neue TOU gegruindet. Insgesamt haben diese jungen Unternehmen hier einen Anteil von 1 bis $2 \%$ an den FuEAufwendungen all derjenigen KMU, die mehr als 100 TDM/Jahr an FuE-Mitteln aufwenden (Kulicke et al., 1993, S. 5). In Ostdeutschland ist der Anteil solcher Neugrïndungen deutlich größer. Neuere Analysen gehen davon aus, daß es gegenwärtig in Deutschland mehr als 50.000 innovative Unternehmen im produzierenden Gewerbe gibt, wovon über die Hälfte eigene FuE durchführen (Harhoff et al., 1996).

Wie in den neuen Bundesländern insgesamt, hat sich auch im Land Brandenburg eine neue Wirtschaftsstruktur herausgebildet. Gegenwärtig gibt es hier etwa 40.000 Unternehmen, davon 2 bis 3\% in der Industrie und die übergroße Mehrheit im Handwerk (mit durchschnittlich 11 Beschäftigten) bzw. im Handel (mit durchschnittlich 4 Beschäftigten). Besonders auffällig und strukturpolitisch problematisch ist der geringe Anteil von Industrieunternehmen im Vergleich zu den $14 \%$ bezogen auf ganz Deutschland. Von den Brandenburger Industrieunternehmen haben $82 \%$ unter 100 Beschäftigte, allerdings beträgt der Anteil von Unternehmen mit unter 20 Beschäftigten erst 21\%. Der Trend zu kleinen Unternehmensgrößenklassen ist in Brandenburg seit Jahren zu verzeichnen und wie der gesamte wirtschaftliche Anpassungsprozeß noch nicht abgeschlossen.

Angesichts der Bedeutung der kleinen Unternehmen lösen deren verstärkt auftretende krisenhafte Entwicklungsprobleme und die zunehmenden Liquidationsraten in Deutschland wie auch im Land Brandenburg Besorgnis bei den wirtschaftspolitischen Akteuren aus und fordern Aktivitäten heraus. Hierzu zählen die Unterstuitzung von Unternehmensgründungen, die länger andauernde Begleitung und Beratung der Unternehmen in krisengefährdeten Entwicklungsstadien sowie die Qualifizierung des Grüindungs-, Krisen- und Innovationsmanagements.

\section{Konzeptionelle Ansatzpunkte für ein angepaßtes Innovationsmanagement in $\mathrm{KMU}$}

\subsection{Unternehmensfunktion und Innovation}

Das Innovationsmanagement entstand und profilierte sich in erster Linie als Reaktion auf Wettbewerbs-, Wachstums- und Strukturprobleme, denen sich große Unternehmen in der Auseinandersetzung mit der zunehmenden Dynamik und Turbulenz ihrer Umfelder gegenübersahen (Servatius, 1988; Zahn (Hrsg.), 1995). Mit den daraus hervorgegangenen Denkweisen und Managementinstrumenten wird häufig mehr oder weniger stillschweigend undifferenziert so umgegangen, als handle es sich bei allen Unternehmen um gut strukturierte, organisierte und ressourcenstarke Institutionen. Aus dieser Sicht kann man den Standpunkt beziehen, daß mittlere und kleine Unternehmen letztendlich den gleichen Anforderungen im Wettbewerb und hinsichtlich ihres Innovationsverhaltens genügen müssen wie die Großunternehmen. Das Problem des Innovationsmana- gements besteht dann im wesentlichen nur im größer/ kleiner dimensionierten Umgang mit gleichen Sachverhalten und Aufgaben.

Angesichts der überwiegenden Mehrzahl kleiner mittelständischer Unternehmen und ihrer Entwicklungsprobleme, der oft als zu gering befundenen Wirkung von technologie- und wirtschaftspolitischen Fördermaßnahmen und des relativ wenig entwickelten Bedarfs nach Beratungsleistungen bzw. geringer Wertschätzung ihrer Ergebnisse (Kulicke et al., 1993, S. 185 ff), kann man aber auch den Standpunkt vertreten, daß kleine Unternehmen vor spezifische Probleme gestellt sind, die dementsprechende Innovationsaktivitäten und ein angepaßtes Innovationsmanagement erfordern.

Zur Überprifung dieser Annahme und zur Ableitung von Schlußfolgerungen für die Gestaltung eines inhaltlich, methodisch und instrumentell angepaßten Innovationsmanagements sind die Bedingungen und Determinanten des Innovationsverhaltens, insbesondere kleiner technologieorientierter Unternehmen, weiter zu hinterfragen. Folgende Ausgangsfragen sollten neu gestellt und geprüft werden:

- Hängen die Entwicklungsprobleme der kleinen innovativen Unternehmen von besonderen Bedingungen $\mathrm{ab}$, die zu speziellen Anforderungen führen und zu wenig durch den Unternehmer selbst und ihn unterstützende Akteure aus den Umfeldern (Forschungsund Technologiepolitik, Kammern und Verbände, Genehmigungs- und Kontrollbehörden) erkannt und berücksichtigt werden?

- Welche Rolle spielen Ressourcenlimitierungen im Unternehmen für das Innovationsverhalten?

- In welchem Maße sind die Entwicklungsprobleme Mißmanagement und Kompetenzdefiziten im Unternehmen bzw. beim Unternehmer geschuldet?

Nach bisherigem Erkenntnisstand werden den KMU im Vergleich zu großen Unternehmen im allgemeinen bestimmte Stärken bzw. Vorteile wie unbürokratische Organisationsform, flache Hierarchien, kurze Informationswege und rasche Entscheidungsfindung, schnelles Aufspüren von Marktnischen, hohe technische Kompetenz, Motivation und flexibles Reagieren auf spezifische Kundenanforderungen zugesprochen. Gleichzeitig geht man von typischen Schwächen und Nachteilen aus, so z. B. hinsichtlich der multivalenten Nutzung von FuEErgebnissen, der Arbeitsteilung und Spezialisierung im Unternehmen, der Größenordnung in Fertigung und Vertrieb, der Marketinginstrumente und Marktmacht, der Finanzierungsmöglichkeiten für Innovationsprojekte sowie eines professionellen Managements (Pleschak et al., 1994, S. 14).

Allgemeine Stärken und Schwächen deuten zwar auf spezifische Entwicklungsbedingungen und Anforderungen hin, erklären aber nicht schlüssig die Entwicklungsprobleme von KMU und sind auf mögliche Konsequenzen fuir ein angepaßtes Innovationsmanagement kaum geprïft oder vertieft untersucht worden. Ob die genannten generellen Stärken der KMU von ihnen auch tatsächlich spezifisch genutzt werden können, hängt von den konkreten Entwicklungsbedingungen und -phasen der Unternehmen sowie von ihren Kompetenzen ab, Umfeldveränderungen als Chancen zu erkennen und mit hoher 
Lernfähigkeit innovativ und flexibel darauf zu reagieren. Ansatzpunkte für ein tieferes Verständnis des spezifischen Innovationsverhaltens von KMU und ein daran angepaßtes Innovationsmanagement liegen in der Analyse des Zusammenhangs von Unternehmung, Unternehmensumfeldern und Innovation.

Das grundsätzliche Ziel unternehmerischer Aktivität ist die Erstellung eines am Kundennutzen orientierten, vermarktbaren, wettbewerbsfähigen Leistungsangebots mit hohem wirtschaftlichen Ertrag und spuirbarem Beitrag zum Unternehmenswachstum. Die Umsetzung dieser Zielstellung erfordert immer wieder eine neue effiziente Kombination von unternehmensintern verfügbaren und extern beschaffbaren materiellen und immateriellen Ressourcen als Reaktion auf sich verändernde direkte und globale Unternehmensumfelder (Albach u. Albach, 1990, S.V; Kulicke et al., 1993, S. 18).

Eine solche Neukombination der Ressourcen zur effizienten Hervorbringung und Vermarktung von Produkten, Verfahren und Dienstleistungen zur Befriedigung von Kundenbeduirfnissen wird, allgemein der Schumpeterschen Auffassung folgend, als Innovation verstanden (Schumpeter, 1931, S. 100f.).

Die o. g. Umfelder werden komplexer und dynamischer, z. B. durch wechselnde Nachfragen, differenziertere Kundenanforderungen, kürzere Produktlebenszyklen, zunehmende Wettbewerbsintensität, Technologiesprünge, Vernetzung der Technologien, Wertewandel bei Nutzern, Beteiligten und Betroffenen sowie sich ändernde wirtschaftspolitische und rechtliche Rahmenbedingungen.

Die Unternehmen stehen damit vor der Aufgabe, beständig ihre Reaktionsfähigkeit (Flexibilität und Geschwindigkeit) den sich dynamisierenden Umfeldern anzupassen. Die entscheidende Antwort der Unternehmen auf diese Herausforderung ist die Hervorbringung und Vermarktung von Innovationen.

Die Chancen für ein dauerhaft erfolgreiches Innovationsverhalten wachsen, wenn es gelingt, nicht nur technische Produkt- und Prozeßneuerungen zu realisieren, sondern eine integrierte Innovationsantwort hervorzubringen, die Innovationen im Organisations- und Personalbereich des Unternehmens und in seinen Interaktionen mit Umfeldakteuren einschließt. Dies erfordert, die Austauschprozesse innerhalb des Unternehmens und mit seinen Umfeldern neu zu gestalten, wobei diese Gestaltungsprozesse in ihrem Kem Lemprozesse sind.

Die besondere Bedeutung der Umfelder für Innovationen in KMU ergibt sich u. a. aus folgenden Zusammenhängen:

- Viele Innovationsimpulse kommen aus direkten und globalen Umfeldern (z. B. Kundenbeduirfnisse, Konkurrentenverhalten, technologische Entwicklung).

- Umfelder wirken als Selektionsfilter für die Auswahl und Bewertung von Innovationsideen und -projekten (Märkte, technologische Alternativen, Rahmenbedingungen, Wettbewerbsstrategien, Wertewandel).

- Umfelder liefern wichtige Ressourcen und bieten Bedingungen fuir die Hervorbringung und ökonomische Realisierung von Innovationen (Arbeitskräfte, Kapital, Zulieferungen, FuE-Kooperation, Infrastruktur, Absatzmärkte).

\section{2 Determinanten und Entwicklungskontexte des Innovationsverhaltens}

Innovationsverhalten von Unternehmen ist im allgemeinsten Verständnis die spezifische Art und Weise, wie der Prozeß der Neukombination von Ressourcen, Angebot und Nachfrage unternehmensintern und bezogen auf die Umfelder orientiert und realisiert wird.

Das Innovationsmanagement umfaßt all jene strategischen Initiativen und dispositiven operativen Handlungen von Führungs- und Leitungskräften des Unternehmens, die auf die Erhaltung und Erweiterung der Kompetenzen für ein flexibles, effizientes und damit wettbewerbsfähiges Innovationsverhalten gerichtet sind.

Jedes Innovationsverhalten eines Unternehmens ist im Grunde einmalig, ansonsten würde es zu keiner Innovation führen. Die Einmaligkeit ergibt sich weiterhin aus der Vielfalt und Entwicklungsdynamik der Ressourcen, Bedürfnisse und Nachfragen sowie der Vielzahl ihrer Kombinationsmöglichkeiten. Dennoch gibt es Grundmuster des Innovationsverhaltens, die durch die Wirkung bestimmter Einflußfaktoren auf den Innovationsprozeß sowie von Entstehungs- und Entwicklungskontexten der Unternehmen determiniert werden.

Es stellt sich die Frage, wie solche Grundmuster für kleine innovative KMU aussehen, durch welche Kombination determinierender Einflußfaktoren und Kontexte sie geprägt werden und ob sich darauf bezogen ein angepaßtes Innovationsmanagement entwickeln sowie zur Anwendung empfehlen läßt.

Die o. g. Schwächen von KMU sind wesentlich auf Defizite in ihrem Innovationsverhalten zurückführbar. Das heißt, oft gelingt es nur unter Ressourcen-, Zeit- und Ertragsverlusten, die das Innovationsverhalten beeinflussenden internen und externen Faktoren auf neue Weise zu kombinieren (vgl. Abb. 1). Das betrifft z.B. die einseitige oder überhöhte Berücksichtigung einzelner Einflußfaktoren, mangelnde Nutzung von Synergiemöglichkeiten und Fehleinschätzungen des Zeitverhaltens der Faktoren. Zusätzliche Probleme entstehen für kleine Unternehmen oft noch daraus, daß sie einerseits über vergleichsweise wenig strukturierte interne Know-how-Bereiche und andererseits über stark spezialisierte einzelne Know-how-Träger verfügen. Demzufolge müssen sie einen besonders großen Teil der leistungsbeeinflussenden Faktoren durch Austauschprozesse mit Umfeldern realisieren.

Die Frage nach der Grundstrategie für das Innovationsmanagement im Sinne von „make or buy“ fuihrt in kleinen Unternehmen aus den verschiedensten objektiven und subjektiven Gründen häufig zu einem ineffizienten und seine Kompetenzprofile überfordernden „do it yourself " für den Unternehmer.

Die unternehmensinternen das Innovationsverhalten beeinflussenden Faktoren sind in erster Linie durch den Unternehmer und seine Mitarbeiter gestaltbar. Die fuir das Unternehmen relevanten externen Ressourcen werden dagegen durch Umfeldakteure gehalten und entwickelt. Solche externen möglichen Ressourcengeber wie Kunden, Zulieferer, Wettbewerber, FuE-Einrichtungen, Banken, Behörden und Kommunen verfügen uiber wichtige Informationen, materielle und finanzielle Kapazitäten sowie 


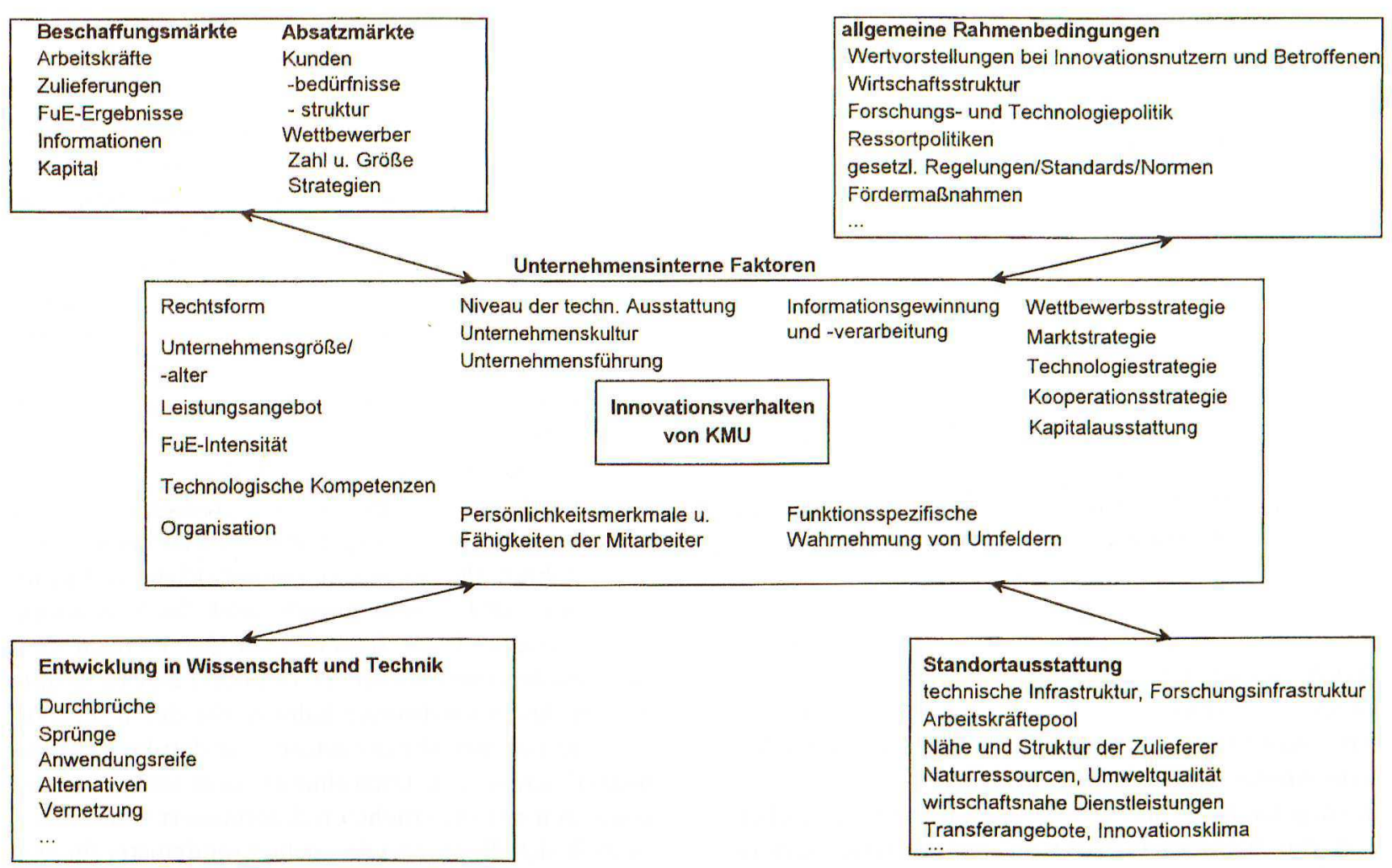

Abb. 1: Interne und externe Einflußfaktoren auf das Innovationsverhalten von KMU

entsprechende Know-how-Träger für die Unterstiitzung von Innovationsprozessen des Unternehmens. Durch ein auf das Innovationsverhalten gerichtetes Management entsprechender Austausch- und Kombinationsprozesse kann das Unternehmen technologische Unsicherheiten (Funktionalität, Stabilität, Alternativen), Marktunsicherheiten (Nachfrage und Wettbewerber) und Planungsunsicherheiten (interne Funktionsschnittstellen, äußere Rahmenbedingungen) reduzieren.

Der Umfang und die Intensität des Austausches, der Kombination und Nutzung von Ressourcen zwischen dem Unternehmen und seinen Umfeldern hängen weiterhin davon $a b$,

- ob, wann und wie sich Unternehmen und Ressourcenhalter wechselseitig wahrnehmen,

- welche Relevanz das Unternehmen den jeweiligen externen Ressourcen und den sie haltenden Akteuren beimißt,

- welche Bereitschaft und Fähigkeit der Unternehmer entwickelt, die Ressourcen anderer Akteure auf seinen Innovationsprozeß zu orientieren, indem er sein Innovationsverhalten mit deren Interessen verknüpft und schließlich

- welches Interesse die externen Ressourcenhalter selbst an einem reziproken Ressourcenaustausch oder einer nutzungsfähigen Ressourcenbereitstellung bezogen auf das Unternehmen haben, welche Relevanz sie diesem ihrerseits für die eigenen Ziele zumessen.

In diesem Prozeß werden allgemeine Ressourcenhalter, auf die sich zunächst einmal viele Unternehmen ähnlichen Profils beziehen können, zu spezifischen Ressourcengebern bezogen auf einen konkreten Innovations- prozeß eines bestimmten Unternehmens.

Die das Innovationsverhalten determinierenden Einflußfaktoren sind für große und kleine Firmen von unterschiedlicher Relevanz. So sind z. B. international agierende Großunternehmen weit weniger auf staatliche Forschungsförderung angewiesen als KMU. Für letztere ist diese Förderung oft ein Erfolgsfaktor für Innovationsprojekte und in bestimmten Entwicklungsphasen von existentieller Bedeutung. Bestimmte staatliche Regulierungen, Genehmigungs- und Kontrollvorschriften können dagegen für großdimensionierte und kapitalintensiv produzierende Pharma-Unternehmen (z. B. Wirkstoffe) von entscheidender Bedeutung sein, weniger aber fuir kleine medizinisch orientierte Unternehmen, die Diagnostika herstellen.

Eigene Untersuchungsergebnisse belegen, daß selbst fuir Unternehmen, die auf dem selben Technologiefeld (hier die Biotechnologie) agieren, die Determinanten des Innovationsverhaltens mit unterschiedlichen Relevanzen belegt und für die Ressourcenkombination genutzt werden. Es deutet sich aber an, daß sich für vergleichbare Faktorkombinationen bestimmte Grundmuster des Innovationsverhaltens herausbilden. Allerdings sind diese Analysen, ähnlich wie die zu den jungen technologieorientierten Unternehmen, nicht mit der primären Orientierung auf die Entwicklung des Innovationsmanagements durchgeführt worden (Große et al., 1995). Hier sind sowohl spezifische Auswertungen bereits vorliegender empirischer Ergebnisse zu Entwicklungsbedingungen innovativer KMU als auch neue, vertiefende Analysen erforderlich.

Das Innovationsverhalten von KMU ist nun nicht nur von einzelnen externen und internen Einflußfaktoren geprägt, sondern auch von komplexeren Entwicklungs- 
kontexten abhängig, die zur Ausprägung unterschiedlicher Muster beitragen (vgl. Abb. 2). Diese Innovationsverhaltensmuster sind letztendlich die spezifische Ausprägung und Kombination der Einflußfaktoren sowie die Art und Weise ihrer Nutzung durch die Unternehmen. Eine besondere Rolle als Kontext spielt der Charakter des Innovationsprozesses (Innovationstyp, -komplexität, -phasen, -zyklen): Einerseits ist er von anderen Kontexten (Branche, Innovationsfeld) in gewisser Weise „historisch" geprägt. Andererseits steht er mit Kontexten, die mit der Unternehmensentwicklung verbunden sind (Entstehungstyp des Unternehmens, Entwicklungsstadium, Wachstumstyp) in besonderer Wechselwirkung.

Innovationen werden durch Unternehmen, aber nicht nur von und in Unternehmen gemacht! Deshalb hängt der Erfolg des Innovationsprozesses zwar in erster Linie vom Innovationsverhalten des Unternehmens ab, aber auch, wie bereits dargestellt, vom Verhalten der Umfeldakteure.

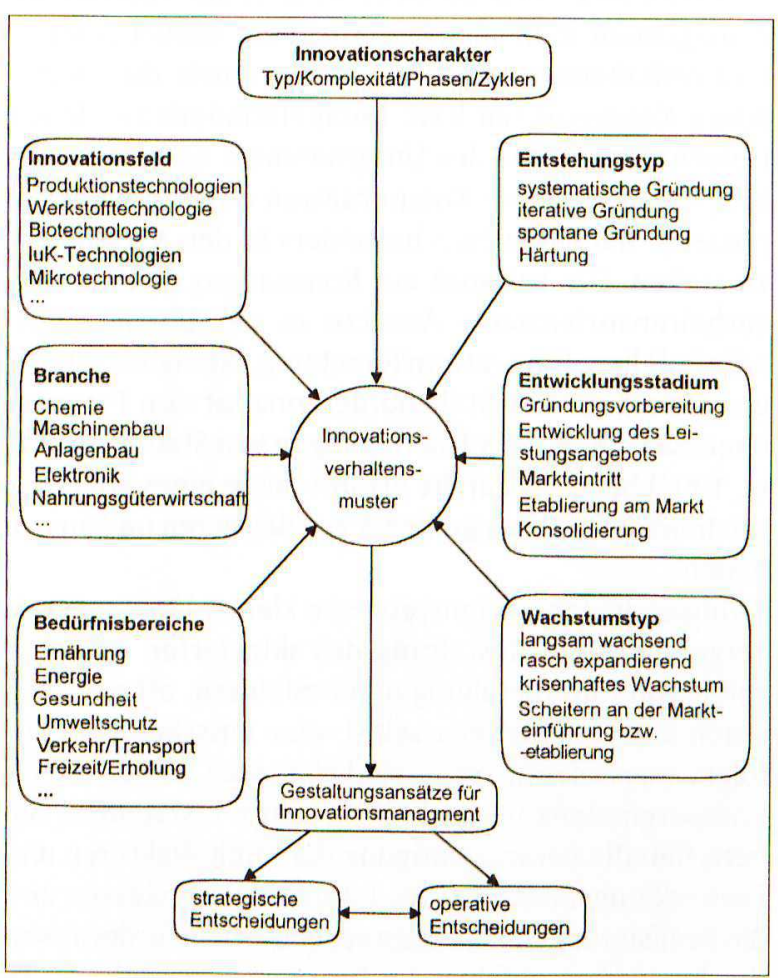

Abb. 2: Kontexte für Innovationsverhaltensmuster

\subsection{Innovationsmanagement als Verhaltensmanagement}

Für die Beantwortung der Frage nach einem angepaßten Innovationsmanagement ist es sinnvoll, die Zusammenhänge zwischen dem Innovationsverhalten des Unternehmens und dem Verhalten der Umfeldakteure als Forschungsgegenstand zu thematisieren und vertieft $\mathrm{zu}$ analysieren.

Folgender Ansatz ist dafuir denkbar: Ein Innovationsverhalten (IV) können - aus dem bereits dargestellten Zusammenhang von Unternehmensfunktion und Innovation begründet - nur Unternehmen entwickeln. Dieses Innovationsverhalten bezieht sich einerseits auf die Gestaltung und Kombination der unternehmensin- ternen Ressourcen und andererseits, bei Limitierungen bzw. Chancen zur Verstärkung eigener Ressourcen, auf den Austausch und die Kombination mit sowie die Nutzung von externen Ressourcen. Diese werden von verschiedenartigen Akteuren mit eigenen Interessen und Zielen sowie entsprechenden Verhaltensweisen gehalten.

Aus der Sicht des Unternehmens haben die Umfeldakteure, deren Ressourcen für seine Ziele relevant sind, ein innovationsrelevantes Verhalten (irV). Das muß nicht bedeuten, daß sich diese Akteure ihrer Relevanz für den Innovationsprozeß des Unternehmens entweder bereits bewußt sind oder - dies vorausgesetzt - auch gewillt bzw. in der Lage sind, ihr Verhalten auf diesen Prozeß zu orientieren. Der Unternehmer bzw. (soweit in kleinen Firmen vorhanden) ein ausdifferenziertes Management stehen nun vor der komplizierten Aufgabe, je nach Relevanzbewertung, im Umgang mit externen Ressourcenhaltern zu erreichen, daß diese ihr Verhalten gezielt auf den unternehmerischen Innovationsprozeß ausrichten und damit aus innovationsrelevantem Verhalten direkt innovationsorientiertes Verhalten (ioV) wird. Das setzt voraus, daß uiber das Innovationsverhalten die unternehmenseigenen Ziele, Ressourcen und zu erwartenden Ergebnisse für die externen Akteure durch für diese vorteilhafte Kommunikations-, Kooperations- und Kombinationsangebote transparent und nutzbar gemacht werden. Hier treffen dann verschiedene Verhalten aufeinander, z. B. unterschiedliche Risikoverhalten. Innovationsrelevantes Verhalten läßt sich sicher dann am effizientesten in innovationsorientiertes Verhalten überfuihren, wenn die Chancen für einen beiderseits vorteilhaften Ressourcenaustausch groß sind. Ressourcen, die generell für viele Unternehmen undifferenziert nutzbar sind (z. B. Ressourcen von Behörden) werden in der Regel nicht zu einem dauerhaften, direkt innovationsorientierten Verhalten beim Ressourcenhalter führen.

Indem Umfeldakteure ihr Verhalten zunehmend auf den Innovationsprozeß orientieren (z. B. angepaßte Zulieferungen), beeinflussen sie ihrerseits das Innovationsverhalten des Unternehmens. Damit fordern sie seine Ressourcen und Kompetenzen auf neue Weise heraus, lösen auch neue Zyklen des extern orientierten Ressourcenaustausches mit den gleichen oder neuen Akteuren und damit weitere Prozesse der Bewertung von innovationsrelevantem Verhalten und seiner Überführung in innovationsorientiertes Verhalten aus (vgl. Abb. 3). So kann sich die landesrechtliche Ausgestaltung von Genehmigung und Vollzug auf der Grundlage des Bundesrechts sowie die diesbezügliche Praxis einzelner Länder und Behörden erheblich unterscheiden und das Innovationsverhalten des Unternehmens unterschiedlich beeinflussen.

Nun gibt es aber auch verschiedene Situationen, in denen Akteure ein für das Unternehmen innovationsrelevantes Verhalten haben, der Unternehmer aber nicht (bezogen auf Konkurrenten) oder zu einem bestimmten Zeitpunkt noch nicht (bezogen auf bestimmte Kundengruppen mit zu spezifischen Anforderungen, weniger leistungsfähige und flexible Zulieferer, Behörden) daran interessiert ist, daß sich daraus ein innovations- 


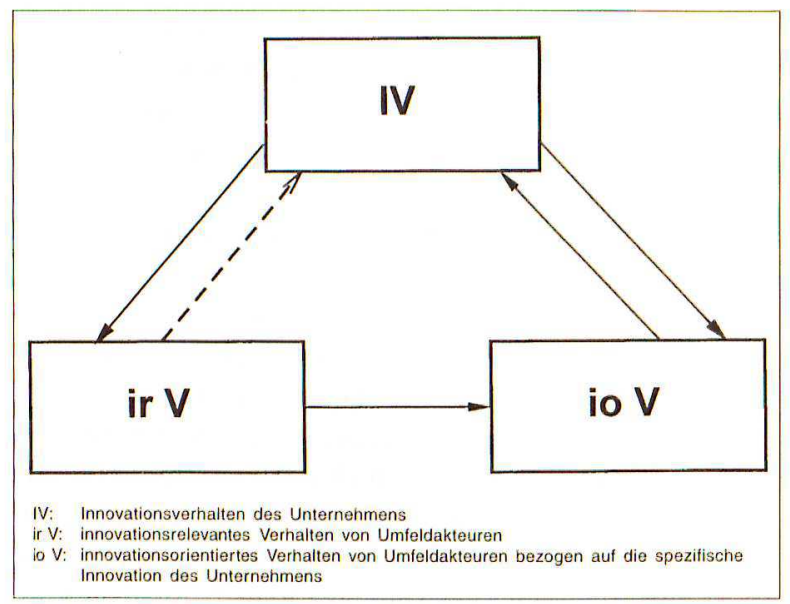

Abb. 3: Akteursverhalten und unternehmerischer Innovationsprozeß

orientiertes Verhalten entwickelt. Er will damit vermeiden, daß z. B. Konkurrenten gezielt eigene innovative Antworten auf das Innovationsverhalten seines Unternehmens suchen, wodurch ihm Zeit- und Kostenverluste entstehen würden.

Das Innovationsmanagement eines Unternehmens muß also dieses komplizierte Wechselspiel von eigenem Innovationsverhalten mit dem innovationsrelevanten bzw. innovationsorientierten Verhaltensweisen der Umfeldakteure gestalten (vgl. Abb. 4). Dabei ist zu berücksichtigen, daß sich diese Prozesse in Abhängigkeit von den determinierenden Einflußfaktoren und den Entstehungs- und Entwicklungskontexten differenziert darstellen. Zu beachten ist auch, daß sich die Anforderungen an das Innovationsmanagement unterscheiden, je nachdem, ob im Interesse des unternehmerischen Innovationsprozesses intern orientiertes Innovationsverhalten zu gestalten, externes innovationsrelevantes Verhalten nur zu nutzen bzw. in innovationsorientiertes Verhalten zu überfuihren ist oder ob es darum geht, bezogen auf bestimmte Akteure diese Überführung zu verhindern bzw. zu verzögern. Auf dem Hintergrund eines solchen Verständnisses von Innovationsmanagement wäre dann z. B. die Frage zu prüfen, welche Vorund Nachteile die oft in „Personalunion“ gleichzeitige

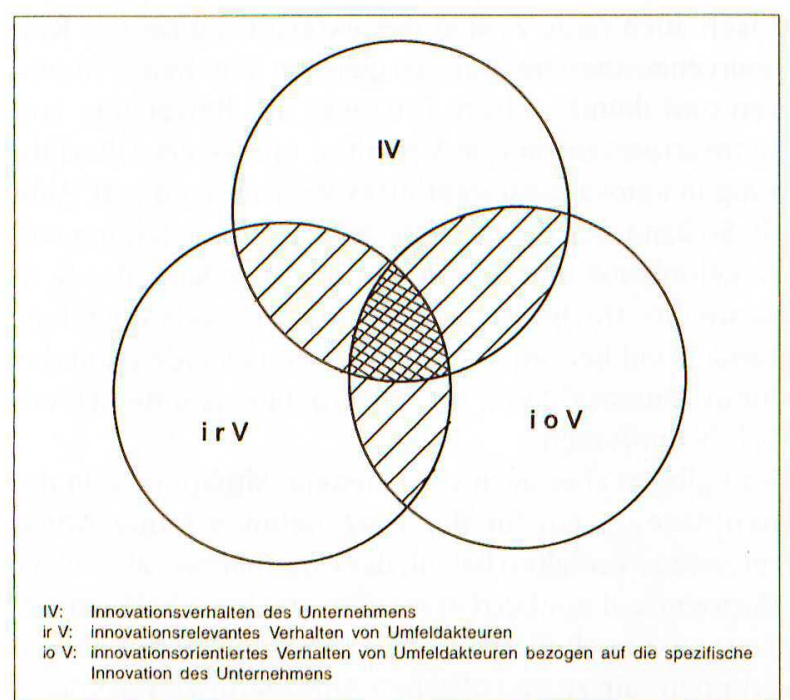

Abb. 4: Innovationsmanagement in kleinen Unternehmen als Verhaltensmanagement
Wahrnehmung verschiedener Promotorenfunktionen (Hauschildt, 1993) im Innovationsprozeß durch den Unternehmer selbst hat. In vertieft untersuchten Fallunternehmen erwies sich ein Zusammenfallen der Funktionen des Macht-, Fach- und Prozeßpromotors als wenig effektiv. Es wäre auch zu untersuchen, unter welchen Bedingungen - bei Kompetenz- und Kapazitätsdefiziten des kleinen Unternehmens - einer oder mehrere Umfeldakteure Funktionen des Prozeßpromotors übernehmen könnten und welche Wirkungen damit verbunden sind.

Viele der kleinen Unternehmen beziehen sich auf Umfeldakteure in einem mehr oder weniger weit gefaßten regionalen Bezugsrahmen. Einerseits wirkt das innovative Milieu einer Region auf das Innovationsverhalten der Unternehmen sowie auf das Verhalten der Umfeldakteure und andererseits wird es ruickkoppelnd selbst beeinflußt. Dieser regionale Kontext ist für den dargestellten Forschungsansatz ebenfalls zu berïcksichtigen und weiter zu durchdringen.

Hier handelt es sich um einen Ansatz, das Innovationsmanagement in kleinen Unternehmen stärker akteursund verhaltensorientiert anzulegen sowie die spezifischen Kompetenzen bzw. Kompetenzdefizite kleiner Unternehmen bzw. des Unternehmers selbst und die Möglichkeiten ihrer Kompensation durch Austauschprozesse mit Umfeldern besonders in den Mittelpunkt zu stellen. Das hat auch zur Konsequenz, vorhandene verhaltensorientierte Ansätze in der Betriebswirtschaftslehre, Innovationsforschung, Sozialforschung u.a., wie z. B. das Stakeholderkonzept von Freeman (Freeman, 1984) oder Überlegungen von Staehle (Staehle, 1991) bezogen auf die Erfordernisse eines angepaßten Innovationsmanagements zu adaptieren und auszubauen.

Erfolgreiche Innovationsprozesse kleiner Unternehmen zeigen, daß die Gestaltung des skizzierten Wechselspiels von Unternehmung und Umfeldern, oft getragen durch den Unternehmer selbst, eine entscheidende Erfolgsvoraussetzung ist. Es sind aber auch immer wieder Kompetenzdefizite auf beiden Seiten feststellbar. Sie betreffen die Berücksichtigung der Einflußfaktoren und Entwicklungskontexte des Innovationsprozesses und die Realisierung eines entsprechend den Erfordernissen der Innovation aufeinander bezogenen Verhaltens. Es bedarf eines längeren Such- und Selektionsprozesses, der vielfach noch sehr ineffizient verläuft, bis sich schließlich relativ stabile und erfolgversprechende Umfelder fuir das Unternehmen herausgebildet haben (vgl. Abb. 5). Dieser Selektionsprozeß kann durch ein bewußt verhaltensorientiertes Innovationsmanagement unterstuitzt werden, indem den in Abbildung 4 dargestellten Schnittflächen durch iteratives Vorgehen besondere Aufmerksamkeit gewidmet wird. Die Schnittfläche aller drei Verhaltensbereiche steht dann gewissermaßen für das letztlich erreichte relativ stabile Umfeld eines Unternehmens, mit dem es seine eigenen Ressourcen kombiniert hat, wie im konkreten Fall in Abbildung 5 gezeigt.

Wie wichtig die differenzierte Betrachtung der o. g. Zusammenhänge für das Vorgehen des Unternehmens ist, hat sich bei eigenen Untersuchungen darin geäu- 


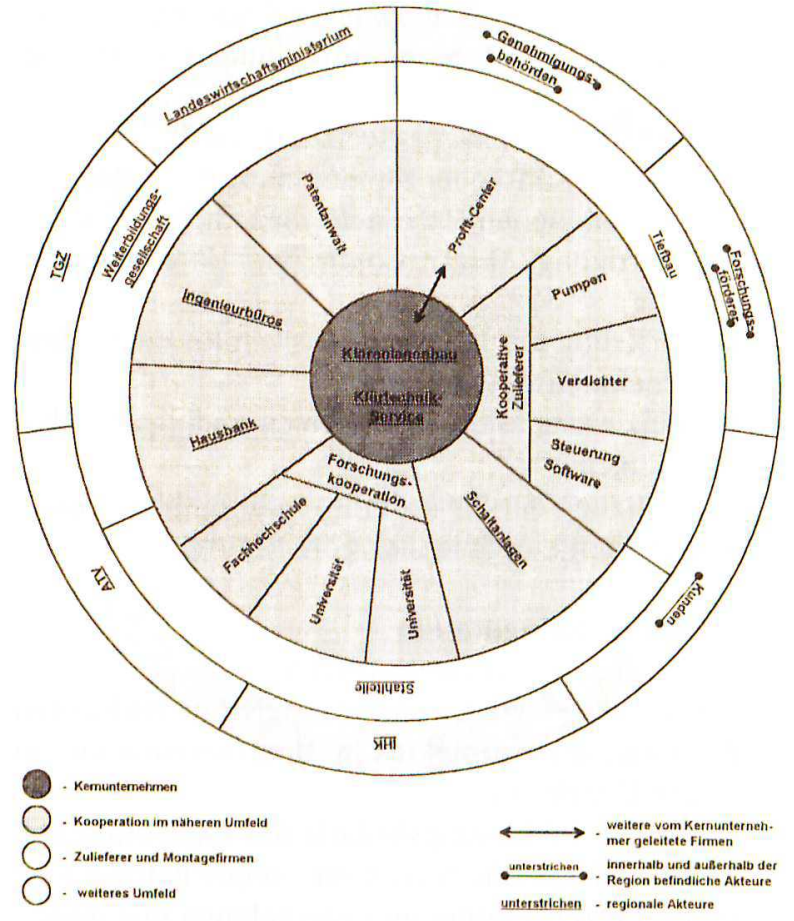

Abb. 5: Anlagenbau-Unternehmen und sein Umfeld auf dem Gebiet „,biologische Abwasserbehandlung"

ßert, daß selbst innerhalb des gleichen Innovationsfeldes agierende Unternehmen je nach Relevanz und Ausprägung der Einflußfaktoren und Entwicklungskontexte im Vergleich zu der dargestellten Abbildung unterschiedlich komplexe und strukturierte Unternehmensumfelder aufgebaut haben, die wiederum ein spezifisches verhaltensorientiertes Innovationsmanagement erfordern. Diese Umfelder führen dann auch zu differenzierten Anforderungsprofilen an die Unternehmen (vgl. Abb. 6).

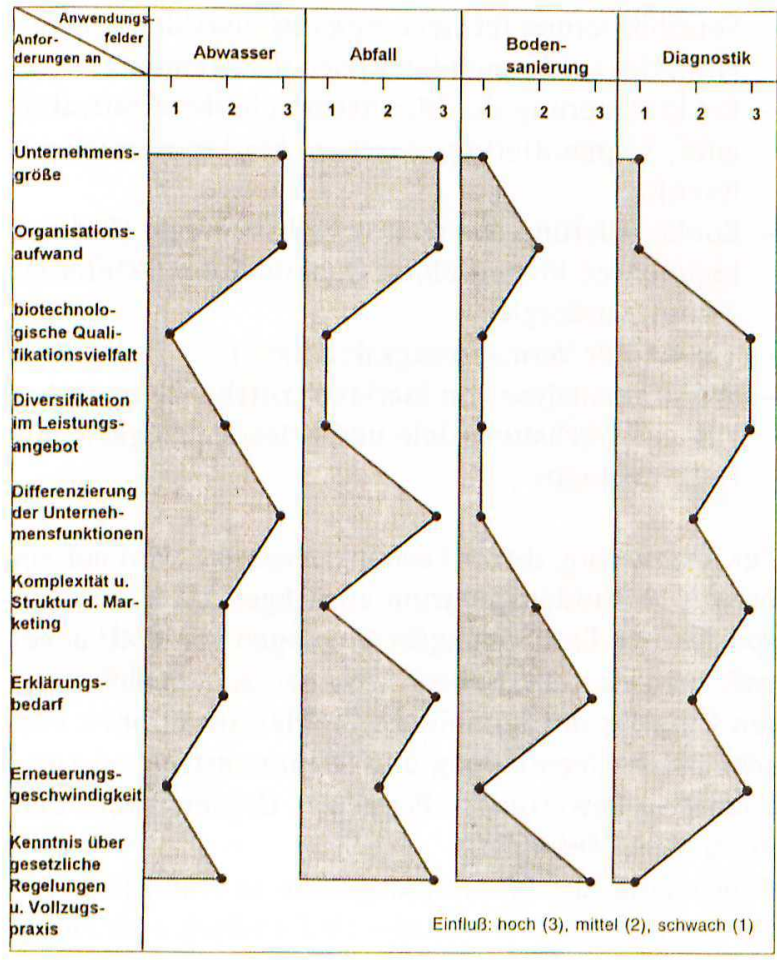

Abb. 6:

Anforderungsprofile für Biotechnologie-Unternehmen auf Anwendungsfeldern
Eine Innovation als wettbewerbsfähige Antwort auf veränderte Umfeldanforderungen gelingt nur dann, wenn die Lerngeschwindigkeit des Unternehmens größer ist als die Geschwindigkeit der Veränderungen in den Umfeldern. Innovationsverhalten beinhaltet deshalb immer gleichzeitig das Verlernen alter und das Erlernen neuer Verhaltensmuster.

\section{Ansatzpunkte für die praktische Unter- stützung des Innovationsmanagements}

Die charakterisierten Probleme können Unternehmen in der Regel nur in einem komplizierten Prozeß und allein selten ohne Entwicklungsverluste lösen. Damit stellt sich die Aufgabe, das Management dabei kontinuierlich begleitend zu unterstiitzen.

Im Land Brandenburg, wie in den neuen Bundesländern insgesamt, kommt hinzu, daß die neugegrindeten, i.d.R. höchstens fünf Jahre alten Unternehmen, kaum über betriebswirtschaftlich und wirtschaftsstatistisch aussagekräftige Vergangenheitsdaten verfuigen, was ihnen einen Zugang zum Innovationsmanagement iiber Lernprozesse aus eigener Erfahrung erschwert. Dies bedeutet aber auch, daß meist noch keine so verfestigten Denk- und Verhaltensmuster ausgeprägt sind, die neues Lernen behindern (Dierkes u. Hähner, 1991). Damit sind Voraussetzungen angesprochen, die auf einen objektiven Bedarf nach Beratung und Begleitung von Innovationsprozessen und Unternehmensentwicklungen hindeuten.

Ausgehend von einem derartigen Innovationsmanagement-Verständnis können angepaßte Beratungs- und Begleitungsangebote fuir kleine und mittlere innovierende Unternehmen entwickelt werden. Zu beachten ist dabei, daß es zahlreiche Aufgaben gibt, die nicht durch managementbegleitende Maßnahmen von außen beeinflußbar sind.

Unabhängig davon, wie schnell es gelingt, die oben dargestellten Ansatzpunkte zu einem schliissigen und operationalisierbaren Forschungskonzept zu entwickeln und in der Praxis zu überpruifen, kann man nicht darauf warten, bis damit erzielte und gesicherte Ergebnisse vorliegen. Parallel dazu muß versucht werden, kleinen innovationsorientierten Unternehmen bei Bedarf wirksame Unterstuitzung bei der erfolgreichen Gestaltung ihrer Innovationsprozesse und Unternehmensentwicklung zu geben. Allerdings sollte man dabei soweit wie möglich bereits vorhandene Kenntnisse und Erfahrungen bezogen auf die spezifischen Wirkungen der Einflußfaktoren und Entwicklungskontexte für eine differenziertere Beratung und Begleitung nutzen und gleichzeitig gewonnene Erkenntnisse im Rahmen neuer Forschungsansätze zu KMU berüicksichtigen. Voraussetzungen dafuir sind, daß die spezifischen Inhalte und Wirkungen der Faktoren, die in den unterschiedlichen Innovationsphasen bzw. Entwicklungsstadien von Unternehmen besonders relevant sind, erkannt und beriicksichtigt werden.

Beispielsweise interessiert das Kundenbedürfnis in frühen Phasen zunächst im Sinne einer Information über den Problemlösungsbedarf, in späteren Phasen dagegen eher im Sinne konkreter Anforderungen an die Funktionen und Leistungsmerkmale sowie der Nutzenspoten- 
tiale für den Kunden (Kosten, Qualität, Zeit). Technologische Entwicklungen werden in frühen Phasen mehr unter dem Aspekt von Technologiebeobachtungen, später zunehmend unter dem von Alternativenbewertungen betrachtet.

Bezogen auf die spezifischen Anforderungen der Innovationsphasen Problemwahrnehmung, Ideengenerierung, Ideenselektion, Implementation und Markteinfuihrung sollen die Unternehmen bei der Ermittlung des Bedarfs an Informationen, Kommunikation und Kooperation und beim Abbau diesbezuiglicher Defizite unterstuitzt werden durch die Bereitstellung von Informationen, Wissen und Methoden für Such-, Selektions-, und Handlungsstrategien. Insbesondere geht es darum, daß die Unternehmen ihre vielfältigen Lernprozesse ruickkoppelnd auf einzelne Innovationsphasen und bezogen auf die Flexibilisierung ihres Innovationsverhaltens wirksamer nutzen.

Unterscheidbar sind Maßnahmen zur Fundierung strategischer Entscheidungen im Hinblick auf die Unternehmensziele und die Ressourcenentwicklung (z. B. Überprüfung grundlegender Prämissen der Wachstums-, Wettbewerbs-, Innovationsstrategie auf Realitätsnähe, Überprifung des Entwicklungsfortschritts und des Ressourceneinsatzes) und operativer Entscheidungen (z. B. Organisationsformen, Finanzierungsformen, Kapitalbeschaffung, Stimulierungsinstrumente, Marketinginstrumente) (Kulicke et al., 1993, S. 208f).

In einzelnen Innovationsphasen nehmen auch die Austauschprozesse mit den Umfeldern und den diese repräsentierenden Akteuren spezifische Formen an. Hier können Hilfestellungen beim Aufbau von Innovationsnetzwerken und bei der Moderation spezifischer Kommunikationsprozesse zwischen Akteuren mit unterschiedlichen Funktionen und Interessenlagen angeboten werden. Im folgenden werden für die verschiedenen Phasen des Innovationsprozesses spezifische Leistungsangebote zur Unterstiitzung des Innovationsmanagements in KMU aufgeführt.

\section{Phase der Problemwahrnehmung}

- Unterstuitzung beim Vorfeldmarketing zur Abschätzung von Nachfragetrends

- Strukturierung innovationsrelevanter Problemlagen in Regionen, Branchen und Anwendungsbereichen

- Analyse von Forschungstrends und -durchbrïchen sowie Technologiemonitoring

- Analyse von Innovationszyklen

- Ermittlung von Wettbewerbsstrategien der Konkurrenten

- Unternehmensgründung

\section{Phase der Ideengenerierung}

- Selektion von Ideenträgern und Erschließung von Zugängen

- Vorschläge für kreative Organisationsstrukturen

- Entwicklung von Anreizsystemen, die das Innovationsverhalten fördern

\section{Phase der Ideenselektion}

- Vereinbarkeit der Innovationsideen mit den Wachstumszielen des Unternehmens
- Abschätzung von Nachfrageentwicklung und -umfang

- Technologiebewertungen (Anwendungsreife, Alternativen)

- Einschätzung der Wettbewerbsintensität

- Analyse der Konsequenzen der Innovationsideen für die verschiedenen Unterneh- mensfunktionen wie FuE, Fertigung, Absatz, Controlling, Unternehmensfuihrung

- Einschätzung der Innovationsrisiken für die weitere Unternehmensentwicklung

- Identifizierung relevanter Normen und gesetzlicher Regelungen

- Einschätzung der Finanzierungsmöglichkeiten und konditionen einschließlich Fördermittel

\section{Phase der Implementation}

- technische Machbarkeitsstudien

- Abschätzung sozialer und ökologischer Wirkungen des Innovationsprojektes im Unternehmen und in seinen Umfeldern

- Analyse des Erklärungsbedarfs der Innovation und Suche nach spezifischen Formen seiner Befriedigung (Akzeptanzerzeugung im Unternehmen und außerhalb)

- Beratung zu Genehmigungs- und Zulassungsverfahren (Behördenmarketing)

- Unterstuitzung beim Schnittstellenmanagement (im Unternehmen und zu Umfeldern)

- Sensibilisierung fuir das rechtzeitige Ermitteln von veränderten Kundenanforderungen an die Funktionen und des Kundennutzens der Innovation

- erste Abschätzung von Markteintrittsbarrieren und Entwicklung von Maßnahmen zu ihrer Reduzierung

- Kommunikations- und Kooperationsstrategien für FuE, Zulieferer und weitere Umfeldakteure

\section{Phase der Markteinführung}

- Sensibilisierung für neue Innovationszyklen als eine Form des vorbeugenden Krisenmanagements

- Konkretisierung der Marktstrategie (Marktattraktivität, Segmentierung, Nischen, Marktpotentialanalysen)

- Konkretisierung der Wettbewerbsstrategie (Technologieführer, Kostenfuihrer, Qualitätsfuihrer, Differenzierungsstrategie)

- Timing der Vermarktungsaktivitäten

- Wirkungsanalyse von Markteintrittsbarrieren in bezug auf Wachstumsziele und Krisenanfälligkeit des Unternehmens

Zur Realisierung dieser Leistungsangebote wird auf ein breites Methodenspektrum zurückgegriffen, das den spezifischen Entwicklungsbedingungen von KMU angepaßt werden muß. Neben Methoden zum qualifizierteren Umgang mit komplexen Problemsituationen einschließlich Ideenfindung und Suchraumstrukturierung umfaßt es Bewertungs-, Planungs-, Organisations- und Controlling-Methoden.

Gleichzeitig sind Leistungsangebote zu entwickeln, die die verschiedenen relevanten Umfeldakteure zu einem spezifischen und wirksamen innovationsorientierten Verhalten befähigen. 
Für die Umsetzung des hier in Ansätzen skizzierten Forschungskonzepts mit Beratungsoptionen bezogen auf die Innovationsprobleme von KMU ist eine Verkniipfung von Know-How aus der Innovationsforschung, Betriebswirtschaftslehre und naturwissenschaftlich-ingenieurtechnischen Bereichen erforderlich. Die Technische Fachhochschule Wildau verfuigt über entsprechende Potentiale, die auch in dieser Richtung bereits verschiedene Aktivitäten entwickelt haben.

Wie bereits weiter oben angedeutet, gibt es wichtige Querverbindungen zwischen dem Innovations-, Gründungs- und Krisenmanagement. Die Notwendigkeit und der zu erwartende Nutzen, die Potenzen dieser Managementbereiche stärker miteinander zu verbinden, ergibt sich aus der ihnen gemeinsamen Orientierung auf die Austauschprozesse zwischen dem Unternehmen und seinen Umfeldern. Umfeldfaktoren wirken als Situationsvariable prägend auf die Ausgangsbedingungen der Unternehmensgründung und gleichzeitig als Prozeßvariable für die Unternehmensentwicklung (Kulicke et al., 1993, S. 139).

\section{Anmerkungen}

1 Der hohe Anteil in Ostdeutschland erklärt sich vor allem aus dem radikalen Abbau der Industrieforschung, von 85.000 FuEBeschäftigten 1989 auf ca. 16.000 (Meske, 1993) und dem Umbau der universitären und außeruniversitären Forschungslandschaft, mit der Freisetzung von etwa 50\% von Wissenschaftlern (Gläser et al., 1995). Diese Mitarbeiter in FuE gründeten oft selbst kleine Unternehmen oder fanden hier Beschäftigung (Gläser et al., 1995). Je nach Technologiefeld und Wirtschaftsbranche weisen diese Prozesse spezifische Züge auf (Voß et al., 1992).

2 FuE-Intensität wird gemessen als Anteil des FuE-Personals an den Gesamtbeschäftigten bzw. Anteil der FuE-Aufwendungen am Umsatz des Unternehmens

3 Unternehmen mit 1-19 Beschäftigten: 35\% FuE-Personalintensität in Ostdeutschland und 18\% in Westdeutschland; Unternehmen mit 20-49 Beschäftigten: $17 \%$ und $8 \%$

4 Höherwertige Technik umfaßt Produktgruppen mit einem Anteil von FuE-Aufwendungen von 3,5-8,5\% am Umsatz.

5 Spitzentechnik umfaßt Produktgruppen mit einem Anteil von FuEAufwendungen mit mehr als $8,5 \%$ am Umsatz.

6 Unspezifische Existenzgründerförderungen; Grïnder- und Technologiezentren; FuE-Personalkostenzuschüsse, Indirektspezifische Projektförderung; Technologietransferstellen; Innovationsassistenten-Programme; Modellversuch „Technologieorientierte Unternehmensgruindungen" $u$. a.

\section{Literatur}

Albach, H.; Albach, r. (1990): Das Unternehmen als Institution. Rechtlicher und gesellschaftlicher Rahmen; eine Einfuihrung. Berlin 1990

Bea, F. X.; Haas, J. (1994): Möglichkeiten und Grenzen der Früherkennung von Unternehmenskrisen; in: Wirtschaftswissenschaftliches Studium, Zeitschrift für Ausbildung und Hochschulkontakt, Heft 10, Oktober 1994, S. 486-491

BMWi (1994): Standort Deutschland. Auftrag Zukunft; hrsg. vom Bundesministerium für Wirtschaft, Bonn 1994

Dierkes, M.; Hähner, K. (1991): Sozioökonomischer Wandel und Unternehmensleitbilder. Ein Beitrag zur Untersuchung der Wahrnehmungsprozesse und Reaktionsweisen von Unternehmen auf Umfeldanforderungen. Wissenschaftszentrum Berlin für Sozialforschung, FS II 91-108

Feser, U. (1995): Krisenmanagement; in: Gründungsplanung und Gründungsfinanzierung. Dieterle, W. K. M.; Winckler, E.

\section{M. (Hrsg.). München 1995}

Freeman, C. (1984): Strategic Management. A Stakeholder Approach. Boston/Mass. 1984

Grünbuch (1995): Grïnbuch zur Innovation; vorgelegt von der Europäischen Gemeinschaft. Brüssel 1995

Gläser, J.; Melis, C.; Puls, K. (1995): Durch ostdeutsche WissenschaftlerInnen gegründete kleine und mittlere Unternehmen; Wissenschaftszentrum für Sozialforschung Berlin, P 95-403

Große, U.; Hartmann, F; Voß, B.; Voß, R. (1995): Marktpotentiale und Unternehmensanforderungen für mittelständische Unternehmen in Mecklenburg-Vorpommern und Brandenburg im Zuge der Anwendung von Biotechnologien; Untersuchung des Instituts für Regionale Innovationsforschung Berlin im Auftrage des Rationalisierungs-Kuratoriums der Deutschen Wirtschaft e.V.; Kurzfassung. Eschborn 1995

Hauschildt, J. (1993): Innovationsmanagement. Müinchen 1993 Harhoff, D.: Licht, G. et. al. (1996): Innovationsaktivitäten kleiner und mittlerer Unternehmen. Schriftenreihe des ZWE, Bd. 8, 1996

Kaiser (1996): Struktur und Entwicklung der Unternehmensgröße in Deutschland. Vortrag von Kaiser, Leiter des Instituts fuir Mittelstandsforschung Bonn, gehalten am 23.3.1996 auf dem Workshop „Innovation und Unternehmertum“ in Berlin Kulicke, M. et al. (1993): Chancen und Risiken junger Technologieunternehmen. Heidelberg: Physika-Verlag 1993, S. If.

Meske, W. (1993): Die Umgestaltung des ostdeutschen Forschungssystems; Wissenschaftszentrum fiir Sozialforschung Berlin, P 93-401

Meyer-Kramer, F. (1889): Der Einfluß staatlicher Technologiepolitikk auf industrielle Innovationen. Baden-Baden 1989 Penzkofer, H. (1992): Innovationsaktivitäten in den neuen Bundesländern; in: Ifo-Schnelldienst (1992), Nr. 15

Pleschak, F; Sabisch, H.; Wupperfeld, U. (1994): Innovationsorientierte kleine Unternehmen, Wie sie mit neuen Produkten neue Märkte erschließen. Wiesbaden: Gabler 1994 Schumpeter, A. (1931): Theorie der wirtschaftlichen Entwicklung - Eine Untersuchung uiber Unternehmergewinn, Kapital, Kredit, Zins und Konjunkturzyklus. 3. Auflage, Leipzig 1931 Servatius, H.-G. (1988): New venture management. Wiesbaden 1988

Staehle, W. (1991): Management - Eine verhaltenswissenschaftliche Perspektive. 6. Auflage, München 1991

Staudt, E. (1996): Die Kompetenz zur Innovation fördern und stärken; in: Arbeitgeber 5/48 - 1996, S. 138-141

SV Wissenschaftsstatistik (1995): Forschung und Entwicklung in der Wirtschaft; FuE-INFO Dezember 1995, SV-Wissenschaftsstatistik GmbH Essen

Voß, R.; Brandt, M.; Hartmann, F; Schiele, K. (1992): Ostdeutsche Biotechnologie im Umbruch; Schriften des Instituts für Regionale Innovationsforschung. Berlin 1992

Wölfing, M. (1994): Was leisten Forschung und Entwicklung zur Erhöhung der Wettbewerbsfähigkeit? In: Herausforderung Ostdeutschland; Pohl, R. (Hrsg.); Institut für Wirtschaftsforschung Halle, 1995

Zahn, E. (Hrsg.) (1995): Handbuch Technologiemanagement. Stuttgart 1995

\section{Verfasser}

\section{Prof. Dr. phil. Rainer Voß}

Technische Fachhochschule Wildau Fachbereich Betriebswirtschaft/Wirtschaftinformatik Tel. (0 33 75) 507-213

Für wertvolle Hinweise nach der kritischen Durchsicht des Manuskripts danke ich meinen Mitarbeitern im Institut für Regionale Innovationsforschung, Dr. Martina Brandt, Dr: Ulla Große und Dr. Frank Hartmann 